Recepción: 22/06/2018

Aceptación: 19/07/2018

Publicación: 20/09/2018

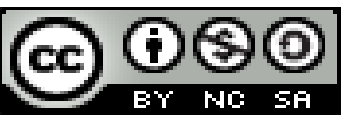

Ciencias sociales y políticas

Artículo de investigación

\title{
Mecanismos de la participación ciudadana en la gestión de los Gobiernos Autónomos descentralizados de los Cantones Bolívar, Tosagua y Pichincha de la Provincia de Manabí
}

\section{Mechanisms of citizen participation in the management of the decentralized Autonomous Governments of the Bolívar, Tosagua and Pichincha cantons of the Province of Manabi}

\section{Mecanismos de participação cidadã na gestão dos governos autônomos descentralizados dos cantões de Bolívar, Tosagua e Pichincha da Província de Manabí}

Rossana Dolores Toala-Mendoza ${ }^{\text {I }}$
amelyta2014@hotmail.com

Baly Ilovasky Vera-Montenegro II

balyvera@gmail.com

Correspondencia: amelyta2014@hotmail.com

\footnotetext{
I Magíster en Gerencia Educativa, Magíster en Auditoria de Gestión de la Calidad, Diploma Superior en Auditoria de Gestión de la Calidad, Licenciado en Contabilidad y Auditoría Contador Público Auditor, Docente Escuela Superior Politécnica Agropecuaria de Manabí Manuel Félix López, Manabí. Ecuador.

II Doctor en Administración, Magíster en Administración de Empresas, Licenciado en Contabilidad y Auditoría, Contador Público Autorizado, Docente Escuela Superior Politécnica Agropecuaria de Manabí Manuel Félix López, Manabí. Ecuador.
} 


\section{Resumen}

El objetivo de esta investigación fue conocer los mecanismos existentes de la participación ciudadana en la gestión de los Gobiernos Autónomos Descentralizados de los cantones: Bolívar, Tosagua y Pichincha de la provincia de Manabí, se utilizaron los métodos de investigación: inductivo, deductivo y descriptivo que permitieron concretar la situación actual de las instituciones. Así mismo se utilizó la recopilación de información bibliográfica que ayudó a sustentar el tema de investigación. Se realizaron entrevistas a los actores principales en los departamentos de Gestión Social y Participación Ciudadana la que fundamentó la investigación, así como también las encuestas con una muestra de 100 personas, la que permitió determinar la incidencia de los mecanismos de participación ciudadana en el desarrollo de la gestión municipal. Las políticas regulatorias se basan en el artículo 100 de la Constitución de la República estipulando que se conformarán instancias de participación, integradas por autoridades electas, representantes del régimen dependiente y de la sociedad según cada nivel de gobierno, los mecanismos que se aplican son las veedurías, la silla vacía, asambleas cantonales, se desarrollan con los actores sociales que son los líderes cantonales, de esta manera se logra que cumplan con las obras que propone esta institución pública.

Palabras Claves: Participación ciudadana; quinto poder; control social; mecanismos; asambleas cantonales.

\section{Abstract}

The objective of this research was to know the existing mechanisms of citizen participation in the management of the decentralized autonomous governments of the cantons: Bolivar, Tosagua and Pichincha in the province of Manabí, used methods of Research: inductive, deductive, descriptive they enabled to realize the current situation of the institutions. Likewise used the compilation of bibliographic information that helped to sustain the research topic. Interviews were conducted with key players in the departments of Social management and citizen participation who founded the research, as well as the surveys with a sample of 100 people, which allowed to determine the incidence of the mechanisms of citizen participation in the development of municipal management. Regulatory policies are based on article 100 of the Constitution stipulating that shall conform instances of participation, composed of elected officials, representatives of the dependent regime and society according to each level of Government, mechanisms that apply are 
the oversight, the empty chair, cantonal assemblies, are developed with the stakeholders who are the cantonal leaders, this way is achieved to fulfill the works proposed by this public institution. Keywords: Citizen participation; fifth power; social control; mechanisms; cantonal assemblies.

\section{Resumo}

O objetivo desta pesquisa foi compreender os mecanismos de participação dos cidadãos na gestão dos governos autônomos de cantões: Bolívar, Tosagua e na província de Pichincha de Manabi, foram utilizados métodos de pesquisa: indutivo, dedutivo e descritivo do que eles permitiram especificar a situação atual das instituições. Da mesma forma, foi utilizada a coleta de informações bibliográficas que ajudaram a sustentar o tema da pesquisa. as entrevistas principais atores foram realizados nos departamentos de Gestão Social e Cidadania que assenta a investigação, bem como pesquisas com uma amostra de 100 pessoas, o que nos permitiu determinar a incidência de mecanismos de participação dos cidadãos no desenvolvimento gestão municipal. políticas regulatórias se baseiam no artigo 100 da Constituição da República que estipula que os organismos de participação, composto por representantes eleitos, representantes do regime dependente e da sociedade em todos os níveis de governo será formado, os mecanismos que se aplicam são as veedurías, a cadeira vazia, as assembleias cantonais, são desenvolvidas com os atores sociais que são os líderes cantonais, conseguindo-se, assim, cumprir os trabalhos propostos por essa instituição pública.

Palavras-chave: Participação cidadã; quinto poder; controle social; mecanismos; montagens cantonais.

\section{Introducción}

\section{Introducción al problema}

La incorporación del derecho y principios de la Participación Ciudadana como uno de los temas preponderantes en la Constitución ha tenido como consecuencia el alto grado de motivación de la población, hecho que se ve reflejado en su involucramiento en la planificación, evaluación y control de la política pública, intervención que es de gran importancia en el desarrollo de la sociedad ecuatoriana (Cuenca, 2017).

La Participación Ciudadana tiene su génesis en la necesidad del ser humano de formar parte como actor de la vida pública del territorio al que se pertenece, mucho tiempo ha tenido que transcurrir para que esa necesidad se convierta en un derecho que le asiste al hombre en la 
sociedad y otro tanto para que sea normado de tal forma que se den todas las aristas para que esa participación sea efectiva y eficaz en todos los niveles del Estado (Cuenca, 2017).

En el Ecuador en las últimas décadas la participación ciudadana ha tomado fuerza con la reforma de la Constitución Política elaborada en el año 2008 en Montecristi y rectificada por los constituyentes, en donde se creó el quinto poder del Estado como menciona la Ley Orgánica Participación Ciudadana (2011), para lo cual es necesario crear espacio de participación ciudadana, en la que las personas puedan integrarse ejerciendo su opinión en los diferentes mecanismos creados con el objetivo de que el usuario sea un miembro activo en la toma de decisiones gubernamentales, teniendo en cuenta que estas actividades de la democracia representativa, constituyen esferas de interacción Estado-sociedad que afianzan la participación como un derecho y principio de gestión de la institucionalidad pública (Santander, 2014).

De esta manera se puede especificar que la participación ciudadana se debe ejercer en las diferentes esferas del sector público, teniendo en cuenta que es un concepto que ya es conocido no se ha desarrollado de manera integral en todos los estamentos del Estado, por lo tanto mediante esta investigación se busca poner en relieve la importancia de la actuación social en las decisiones que se toman en los gobiernos locales, pero para que se pueda desarrollar a cabalidad es importante el compromiso político de las autoridades locales, pero también de los niveles de organización social (Ramón, 2012).

La participación ciudadana no se ha desarrollado cabalmente en los Gobiernos Autónomo Descentralizados, teniendo en cuenta que como se estipula en el 2 artículo 72 de la Ley Orgánica de Participación Ciudadana, las instituciones públicas deben establecer instrumentos que permitan que la ciudadanía se integre de forma individual o colectiva en todos los niveles de gobierno establecidos en la Constitución y la ley, los mecanismo existentes son las audiencias públicas, cabildos populares, silla vacía, las veedurías, los observatorios y los consejos consultivos, la consulta previa, rendición de cuentas, del libre acceso a la información pública (Ley Orgánica Participación Ciudadana, 2011).

\section{Importancia del problema}

La presente investigación nos permite conocer lo que acontece en los Gobiernos Autónomos Descentralizados Municipales de los cantones Bolívar, Tosagua y Pichincha, en lo que guarda relación a la Participación Ciudadana y cómo ésta han repercutido los mecanismos utilizados para el desarrollo social de estos cantones. 
La investigación es importante debido a las distintas formas de participación ciudadana que existen actualmente, en la que se permite involucrar a la población en los diferentes aspectos que caracterizan a la administración pública, en especial en el desarrollo de las diferentes propuestas en mejora de las diferentes localidades y sectores del país, todo esto es debido a la creación del quinto poder que es la ciudadanía.

La Ley Orgánica de Participación Ciudadana en el artículo 72, propone mecanismos que permiten la actuación de las personas en el ámbito gubernamental, para lo cual se han desarrollado instrumentos de participación como son las audiencias públicas, cabildos populares, silla vacía, las veedurías, los observatorios y los consejos consultivos, la consulta previa, rendición de cuentas, del libre acceso a la información pública (Ley Orgánica Participación Ciudadana y Control Social, 2011).

\section{Metodología}

La investigación fue desarrollada en los GADS Municipales de los cantones Bolívar, Tosagua y Pichincha, ubicados en la provincia de Manabí:

Los métodos de investigación utilizados en la investigación fueron:

- Inductivo con el fin de recopilar información inherente a la investigación, con el objetivo de identificar problemas sobre el nivel de participación ciudadana que se ejerce en el sector público.

- Deductivo el mismo que permitió analizar la información obtenida con el fin de escoger la que permita sustentar la investigación.

- Descriptivo, el método que permitió analizar la información una vez obtenidos los indicadores necesarios de la investigación.

- El tipo de investigación utilizado en la investigación es el de campo, el mismo que permite visitar cada una de las instituciones para conocer su situación actual, con la finalidad de observar el nivel de participación ciudadana que se desarrolla en los cantones Bolívar, Tosagua, y Pichincha de la provincia de Manabí.

También se aplicaron entrevistas, las mismas que se realizaron a los funcionarios responsables de los departamentos de Gestión Social y Participación Ciudadana y encuestas, dirigidas a una muestra de 100 personas, actores sociales y ciudadanos que asisten a los departamentos de participación ciudadana por diferentes acciones. 


\section{Resultados}

\section{1. ¿Qué es para usted la participación ciudadana?}

\section{Análisis e interpretación}

En la encuesta dirigida a los ciudadanos de los cantones Bolívar, Tosagua y Pichincha, en cuanto a la pregunta si conocen lo que es la Participación Ciudadana, la mayoría de las personas encuestadas dieron por respuesta que es un derecho con el $46 \%$ en el cantón Bolívar y el $28 \%$ en los cantones Tosagua y Pichincha, mientras que otras escogieron la opción de que es una iniciativa popular con porcentajes $35 \%$ y 30 en los cantones Tosagua y pichincha respectivamente. Cabe recalcar que hay un número considerable de personas que desconocen lo que es la Participación Ciudadana con el 23\% en el cantón Bolívar, el 17\% en Tosagua y el 20\% en el cantón Pichincha.

\section{2. ¿De qué manera usted ha participado en su jurisprudencia cantonal?}

\section{Análisis e interpretación}

En cuanto a la pregunta realizada a los encuestados sobre de qué manera han sido partícipes en su cantón en cuanto a Participación Ciudadana, manifestaron que lo han realizado con el voto, es así como el 60\% de Bolivarenses lo manifiesta, el 28\% de los ciudadanos del cantón Tosagua y el $35 \%$ de Pichincha. Así mismo se puede notar que un 21\% de encuestados del cantón Bolívar no ha participado en actividades referentes a la Participación Ciudadana, el 20\% y 27\% de encuestados tanto de Tosagua como de Pichincha respectivamente, dieron por respuesta la misma opción.

\section{3. ¿Cree usted que la participación ciudadana es?}

\section{Análisis e interpretación}

Según la encuesta realizada a los actores sociales se pudo identificar que el $42 \%$ de Bolivarenses cree que la Participación ciudadana es muy importante para ellos, así como para las personas del cantón Pichincha con el 37\%, mientras que el mayor porcentaje en el cantón Tosagua se refleja en la respuesta "es importante" con el 35\%. Cabe recalcar que existe un 27\% de encuestados del cantón Pichincha que dieron por respuesta que la Participación Ciudadana para ellos es nada importante, lo que se debería tener en cuenta al momento de plantear las estrategias necesarias para dar conocimiento de la importancia que es que se involucren como ciudadanos en los proyectos que beneficien a su comunidad. 
4. ¿Conoce usted, que organismo le compete promover la participación ciudadana en su cantón?

\section{Análisis e interpretación}

En los resultados de la encuesta según el gráfico del cantón Bolívar se puede observar que un $43 \%$ de encuestados no conoce que organismos le competen promover la participación ciudadana en su cantón, lo mismo en el cantón Pichincha con el 40\%, mientras que un 37\% y 34\% de personas de cantón Tosagua dieron por respuesta que es el GAD Municipal y el Concejo de Participación Ciudadana y Control Social es a quienes le competen promover dicha participación.

\section{5. ¿Conoce usted qué proceso ha promovido el GAD Municipal de su cantón?}

\section{Análisis e interpretación}

La encuesta realizada a los actores sociales ha dado por respuesta que no se ha impulsado ningún proceso de Participación Ciudadana, con resultados como el 57\% el cantón Bolívar, el 30\% el cantón Tosagua y el 54\% el cantón Pichincha. Cabe mencionar que en Tosagua el 36\% de encuestados expresó que se han promovido en las Unidades Básicas barriales.

6. ¿Si ha participado en un proceso de participación ciudadana promovido por el GAD Municipal, lo ha realizado?

\section{Análisis e interpretación}

Los encuestados mencionaron no haber participado en ningún proceso de Participación Ciudadana promovido por el GAD Municipal de sus cantones, tales como el 64\% en el cantón Bolívar, el 37\% en Tosagua y el 50\% de Pichincha. Existe un 29\% y 30\% de los cantones Tosagua y Pichincha respectivamente que han participado, pero como delegados.

\section{Entrevistas realizadas a los funcionarios responsables de los departamentos de} participación ciudadana

\section{Entrevista en Gad del Cantón Bolívar}

En la entrevista realzada en el GAD del cantón Bolívar al señor Wagner Ganchoso economista de profesión se pudo determinar que el tema de participación ciudadana se está dando de manera progresiva en los distinto medios de los gobiernos sean cantonales o provinciales, se está dando de una manera poca lenta, pero en todo sentido se está cumpliendo con el marco jurídico.

De alguna manera se estado involucrando desde dentro de casa a los ciudadanos y a los actores principales que son los jefes departamentales del GAD esto es un proceso son de manera dual 
tanto la ciudadanía con los que están al frente del departamento de participación, por no se puede hacer un eje de acción solo dirigido a la ciudadanía si las autoridades si casa adentro no hacemos los procesos de participar.

La participación es de manera directa con los ciudadanos a través de las redes sociales, publicaciones por medio de la prensa radial o por medio de la prensa escrita.

Los proyectos que se llevan a cabo y que se han implementado desde el año 2012 el tema de los presupuestos participativos, un porcentaje del presupuesto de la institución, otro porciento se lo obstruye de manera directa con la ciudadanía se hace un cálculo moderado con la ciudadanía para que ellos hagan conocer sus necesidades básicas y de las que no estén satisfechas en todo el territorio de acuerdo a la administración, también se toma en cuenta a las parroquias tanto Calceta, Quiroga y Membrillo se han beneficiado de este proceso de participación ciudadana a través del presupuesto participativo porque son ellos los que deciden qué obra hacer, cuando hacerla y como hacerla.

Se involucra a los presidentes barriales en estos procesos si están obligados hacerlo por mandato legal y muchos más acá en el cantón Bolívar cuando al 1 de octubre del 2017 se llevan dando la elección obligatoria de una comisión barrial que son unidades básicas de participación ciudadana y por tanto se con lleva con ellos a coordinar sus acciones entre obras y servicios en cada uno de los barrios.

Los temas de participación ciudadana son viables siempre hay que estar de manera coordinada con ellos trabajando, bueno los resultados se calcula que son favorables son buenos porque en todos estos procesos de presupuestos participativos la silla vacía y la rendición de cuentas son evaluados por medio del Consejo de Participación Ciudadana por Campadles, Defensoría del Pueblo, también por la Procuraduría General del Estado.

\section{Entrevista en Gad del Cantón Tosagua}

En la entrevista realzada en el GAD del cantón Tosagua se pudo determinar que la participación es tanto de la ciudadanía como el compromiso con la participación se integran mecanismos y atender las necesidades del ciudadano. El GAD ha realizado obras conjuntamente que van de la mano con la ciudadanía, con y ellos el apoyo de las a autoridades.

Que hubo una reforma si pero no hay una ley de definición social donde se definen algunos puntos muy importante que la ley de participación ciudadana existe ya que nunca antes se había tomado en cuenta en los GADS Municipales, esto es un derecho de todos los ciudadanos dentro 
de las funciones del estado es importante que este poder ciudadano se mantenga pero que no se trate de manipular unos de los aspectos de esta ley es : fortalecer la rendición de cuentas, asambleas cantonales y veedurías públicas.

Es importante conocer que esta ley tenga equidad ya que es uno de los principales temas de la Participación, pero no todos estos aspectos están fortalecidos, pero tenemos que seguir en lucha para que día a día se fortalezcan estos temas de interés público.

El tema principal siempre será la rendición de cuentas, como anteriormente se mencionó que son varios, pero la rendición de cuenta en estos últimos años es el tema más común en la entidad pública del estado, la rendición de cuenta solo aplica para los GAD y EL CUERPO DE BOMBEROS por son entidades públicas autónomas y es allí donde la ciudadana tiene que intervenir y conocer como son manejados dichos temas.

A raíz de la rendición de cuentas se han fortalecidos los consejos barriales, pero con poca participación que se desconocen los artículos de la Participación Ciudadana sí, pero los ciudadanos ignoramos este tema.

En el 2014 el GAD de Tosagua se dio una nueva ordenanza para elegir una nueva asamblea local es ahí donde se eligen comité de los consejos barriales, pero estos siempre van dirigidos por la máxima autoridad, este sistema está conformado por te eje que son: Las autoridades electas, las autoridades básicas que son las que tiene que fortalecer este tema por eso la participación ciudadana estas participaciones pueden ser Local, Parroquial o Provincial.

En el 2017 para conformar la asamblea local se seleccionó a un representante barrial para conformar un gremio para que el cantón tenga voz y voto, para que se lleva a cabo la asamblea del buen vivir que desconocemos que es esto pues muchos de los ciudadanos del cantón lo desconocen, no tanto porque desconocen del tema si no porque muchos de ellos piensan esto es política, pero están equivocados.

Dentro del cantón se han dado muchas obras, donde los ciudadanos en las asambleas cantonales, o en presupuesto participativos han dado a conocer cuáles son las necesidades que tiene en sus barrios o en su comunidad.

Como desarrollamos esto a continuación detallamos obras que si se han dado y obras que factor económico no se han podido ejecutar:

504

Pol. Con. (Edición núm. 25) Vol. 3, No 9, septiembre 2018, pp. 496-508, ISSN: 2550 - 682X 
Cancha de uso múltiple como se desarrolló esto el municipio coloca el material, pero la mano de obra, el terreno donde se ubica la cancha tiene que ser un lugar donde no se corra el riesgo, y si es donado saber que es por voluntad propia, o tener problemas legales.

En la calle Sta. Lucia vía al Tambo aún no se puede dar comienzo a la obra por no contar con los suficientes recursos.

Los cursos vacacionales que fueron solicitados por un grupo de madre y ex reinas del cantón.

Cuales han sido las estrategias para fortalecer la Participación Ciudadana, han sido las asambleas barriales, presupuestos participativos donde se invitan a todos los ciudadanos ya que ellos no quieran asistir por que dicen que el tema es político hay mucha diferencia, en las asambleas realizadas por el GAD siempre se ubican mesas de trabajo para que la ciudadana participe, ya que no es lo mismo participar detrás de un escritorio, a tener que conocer las necesidades del cantón.

\section{Entrevista en el Gad del Cantón Pichincha}

La participación ciudadana es un tema importante que en los últimos años en el Ecuador ha tomado mucha fuerza ya que está en el código vigente de la actual Constitución de la Republica, tiene como finalidad que los ciudadanos y las autoridades tenga un diálogo abierto, esto es un proceso que no será fácil pero que día a día tendrá que fortalecer nadie dice que no ha sido fácil ni difícil este proceso llega varios años en la lucha pero que aún falta mucho por fortalecer a la ciudadanía del cantón Pichincha.

Esto se está dando de varias maneras con la ayuda que nos brinda el gobierno sea esta provincial, cantonal, la Participación Ciudadana en el GAD de Pichincha se está fortaleciendo primero con sus parroquias rurales ya que en la cabecera Cantonal ha sido un poco difícil el trabajo con los ciudadanos, pero estamos en la lucha.

En las oficinas se han estado involucrando desde afuera hacia dentro con los ciudadanos y a los principales jefes de los departamentos del GAD esto es un proceso son de manera que tanto la ciudadanía como los que estamos al frente del departamento de participación ciudadana, estábamos haciendo un argüe trabajo para así desde casa adentro logramos hacer los procesos de participar.

Como logramos llegar a los habitantes para que conozcan acerca de la participación ciudadana desde hace un año más o menos lo venimos haciendo de manera directa con los ciudadanos con la facilidad que nos brindan las redes sociales, publicaciones por medio de pancartas o por boletines. 
Hemos implementados varios programas y proyectos públicos, la programación y ejecución del presupuesto del Estado la inversión y la asignación de los recursos públicos y coordinará las competencias exclusivas entre el Estado central y los Gobiernos Autónomos Descentralizados. Su observancia será de carácter obligatorio para el sector público e indicativo para los demás sectores.

Se involucra a los presidentes barriales en estos procesos si están obligados hacerlo por mandato legal y muchos más acá en el cantón Pichincha que desde al año 2016 se llevan dando la elección de una comisión barrial que son unidades básicas de participación ciudadana.

Los planes de ordenamiento territorial regionales, provinciales y parroquiales se articularán entre sí, debiendo observar, de manera obligatoria, lo dispuesto en los planes de ordenamiento territorial cantonal y/o distrital respecto de la asignación y regulación del uso y ocupación del suelo.

La participación ciudadana es viables siempre hay que estar de manera coordinada con ellos trabajando, para obtener buenos resultados nosotros calculamos que son favorables son buenos porque en todos estos procesos de presupuestos participativos la silla vacía y la rendición de cuentas son evaluados por medio del Consejo de Participación Ciudadana, Defensoría del Pueblo, también por la Procuraduría General del Estado.

\section{Conclusiones}

Los mecanismos de participación ciudadana donde intervienen el mayor número de ciudadanos, son las veedurías, la silla vacía, asambleas cantonales, peticiones que buscan implementar las políticas para el buen vivir, las iniciativas populares planteadas tienen relación con programas de solidaridad, salud y ordenamiento territorial, lo cual es mencionado en el artículo 95 de la Constitución de la República del Ecuador la cual estipula que "las ciudadanas y ciudadanos, en forma individual y colectiva, participarán de manera protagónica en la toma de decisiones, planificación y gestión de los asuntos públicos, y en el control popular de las instituciones del Estado y la sociedad”, así mismo en su artículo 207 menciona que "el Consejo de Participación Ciudadana y Control Social promoverá e incentivará el ejercicio de los derechos relativos a la participación ciudadana, impulsará y establecerá mecanismos de control social en los asuntos de interés público, y designará a las autoridades que le corresponda de acuerdo en la Constitución y 
la ley, la estructura del Consejo será desconcentrada y responderá al cumplimiento de sus funciones".

Las técnicas de investigación propuestas sirvieron para medir el conocimiento de los ciudadanos de los cantones Bolívar, Tosagua y Pichincha en donde se pudo determinar que existe cierto conocimiento por parte de los ciudadanos, pero no existe participación en los procesos de participación ciudadana, en donde deben de intervenir tanto la ciudadanía como los actores y responsables principales de Participación ciudadana.

Se establecieron estrategias para incluir y mejorar el conocimiento en cuanto a Participación Ciudadana para los Gobiernos Municipales de los cantones Bolívar, Tosagua y Pichincha de la provincia de Manabí, ante lo cual es posible concluir que los actores y responsables deben seguir modelos de desarrollo en los que el empoderamiento de la ciudadanía sea un eje primordial para lograr resultados más satisfactorios para los beneficiarios.

\section{Recomendaciones}

Socializar por diferentes medios las ordenanzas existentes que llevan relación con la Participación Ciudadana, de la misma manera que los GADS Municipales incrementen la participación en los diferentes mecanismos de participación a los líderes comunitarios para emprender nuevos proyectos y nutrir de conocimientos a los beneficiarios.

Los actores sociales presentar nuevas propuestas como proyectos apegados a las competencias Municipales que tiene cada Gobierno Autónomo y dar seguimiento para que estos sean atendidos de manera eficaz y en el menor tiempo posible, con la finalidad de solucionar las necesidades más urgentes de las comunidades.

Aplicar las estrategias planteadas para proyectar conocimiento a los ciudadanos sobre lo referente a la participación ciudadana y la manera positiva que influyen en el desarrollo de los proyectos comunitarios, ya que cuando se trabaja de manera integral satisfacen de forma oportuna las necesidades de la ciudadanía, que es el objetivo de toda institución pública.

\section{Agradecimiento}

Los autores del presente trabajo de investigación agradecen a la Escuela Superior Politécnica agropecuaria de Manabí Manuel Félix López, en especial a la Carrera de Administración Pública, a los catedráticos y estudiantes por brindarnos las herramientas necesarias para poder concluir la presente investigación. 


\section{Referencias Bibliográficas}

Cuenca, S. (2017). La participación ciudadana en el GAD de Portoviejo y su incidencia en el desarrollo local. Recuperado el 17 de agosto de 2018, de http://repositorio.ucsg.edu.ec/bitstream/3317/8173/1/T-UCSG-PRE-JUR-DERMD113.pdf

Ley Orgánica de Participación Ciudadana y Control Social. (11 de mayo de 2011). Registro Oficial. Recuperado el 20 de agosto de 2018, de http://www.cpccs.gob.ec/wpcontent/uploads/2017/01/LORGAPARTICI.pdf

Plan Nacional del Buen Vivir. (2014). EJES ESTRATEGICOS. Recuperado el 2018 de agosto de 2018, de http://quito.gob.ec/documents/rendicion_cuentas/AZCH/12ejesdelPNBV.pdf

Ramón, M. (2012). La participación ciudadana en la esfera de lo público. 85. Recuperado el 17 de agosto de 2018

Santander, F. (2014). Indicadores de participación ciudadana y Evaluación de procesos participativos. 25. Recuperado el 17 de agosto de 2018. 